\title{
Mechanism and consequences of the shift in cardiac arginine metabolism following ischaemia and reperfusion in rats
}

\author{
Rolf Schreckenberg ${ }^{1 *}$; Pia Weber ${ }^{1 *}$; Hector A. Cabrera-Fuentes ${ }^{2,6}$; Isabel Steinert ${ }^{1} ;$ Klaus T. Preissner ${ }^{2}$; Péter Bencsik ${ }^{3}$; Márta Sárközy ; \\ Csaba Csonka ${ }^{3,4}$; Péter Ferdinandy ${ }^{3,5}$; Rainer Schulz'; Klaus-Dieter Schlüter ${ }^{1}$ \\ ${ }^{1}$ Physiologisches Institut, Justus-Liebig University Giessen, Germany; ${ }^{2}$ Biochemisches Institut, Justus-Liebig University Giessen, Germany; ${ }^{3}$ Pharmahungary Group, Szeged, Hungary; \\ ${ }^{4}$ Cardiovascular Research Group, Department of Biochemistry, University of Szeged, Hungary; ${ }^{5}$ Department of Pharmacology and Pharmacotherapy, Semmelweis University, \\ Budapest, Hungary; ${ }^{6}$ Kazan Federal University, Department of Microbiology, Kazan, Russia
}

\begin{abstract}
Summary
Cardiac ischaemia and reperfusion leads to irreversible injury and subsequent tissue remodelling. Initial reperfusion seems to shift arginine metabolism from nitric oxide (NO) to polyamine formation. This may limit functional recovery at reperfusion. The hypothesis was tested whether ischaemia/reperfusion translates such a shift in arginine metabolism in a tumour necrosis factor (TNF)- $\alpha$-dependent way and renin-angiotensin system (RAS)-dependent way into a sustained effect. Both, the early post-ischaemic recovery and molecular adaptation to ischaemia/reperfusion were analysed in saline perfused rat hearts undergoing global no-flow ischaemia and reperfusion. Local TNF- $\alpha$ activation was blocked by inhibition of TNF- $\alpha$ sheddase ADAM17. To interfere with RAS captopril was administered. Arginase was inhibited by administration of Nor-NOHA. Long-term effects of
\end{abstract}

Correspondence to:

Prof. Dr. Klaus-Dieter Schlüter

Physiologisches Institut, Justus-Liebig-University Giessen

Aulweg 129, 35392 Giessen, Germany

Tel.: +496419947 212, Fax:+496419947219

E-mail: Klaus-Dieter.Schlueter@physiologie.med.uni-giessen.de

These authors contributed equally to the study. ischemia/reperfusion on arginine metabolism were analysed in vivo in rats receiving an established ischaemia/reperfusion protocol in the closed chest mode. mRNA expression analysis indicated a shift in the arginine metabolism from NO formation to polyamine metabolism starting within 2 hours (h) of reperfusion and translated into protein expression within $24 \mathrm{~h}$. Inhibition of the TNF-a pathway and captopril attenuated these delayed effects on post-ischaemic recovery. This shift in arginine metabolism was associated with functional impairment of hearts within $24 \mathrm{~h}$. Inhibition of arginase but not that of TNF- $\alpha$ and RAS pathways improved functional recovery immediately. However, no benefit was observed after four months. In conclusion, this study identified TNF- $\alpha$ and RAS to be responsible for depressed cardiac function that occurred a few hours after reperfusion.

Financial support:

This study was supported by the Deutsche Forschungsgemeinschaft (grants to K. Preißner, R. Schulz and K.-D. Schlüter within the graduate school "PROMISE" [IRTG1566]) and a grant to K.-D. Schlüter (SCHL 324/7-1).

Received: May 29, 2014

Accepted after major revision: October 28, 2014

Epub ahead of print: December 11, 2014

http://dx.doi.org/10.1160/TH14-05-0477

Thromb Haemost 2015; 113: 482-493

\section{Introduction}

The acute coronary syndrome is one of the leading causes for the development and progression to heart failure $(1,2)$. It can be successfully treated by reperfusion strategies thereby limiting myocardial damage but yet leading to new onset of heart failure. Therefore, a better understanding of the molecular processes in the reperfused post-ischaemic heart is required to improve pharmacological options that prevent the progression of cardiac remodelling to heart failure under such conditions.

Inhibition of the angiotensin converting enzyme (ACE) can positively affect post infarct remodelling specifically if treatment is started after a successful revascularisation. Delayed but not immediate captopril therapy improved cardiac function and increased survival (3-7). This suggests that the renin-angiotensin system (RAS) contributes to early post-infarct remodelling leading to progression to heart failure but not to direct reperfusion-linked injury. The molecular mechanisms that may explain such a behaviour of ACE inhibition in this specific setting are not clear. In an experimental study, captopril improved the bioavailability of nitric oxide (NO) after ischaemia/reperfusion if administered together with arginine (8). This suggests that in the post-ischaemic heart arginine may not be used by nitric oxide synthases (NOS) but by alternative pathways.

In general, an activation of NO-dependent cGMP-linked pathways is considered to improve the early post-ischaemic recovery (9). However, the post-ischaemic myocardium has a characteristic loss of NO-dependent signalling $(10,11)$. This is mainly due to an increased oxidative stress and diminished NO formation rather than the impaired downstream signalling of NO, because administration of $\mathrm{NO}$ donors restores a normal NO-dependent signalling (12). Interestingly, chronic ischaemia leads to endothelial dysfunction in the heart that is independent of arginase activity (13). Therefore, the observed shift in arginine metabolism is specific for 
ischaemia/reperfusion injury. Possible reasons for such a lack of responsiveness are either a down-regulation of NO forming pathways or an activation of pathways that compete with $\mathrm{NO}$ formation for the same substrates.

Arginine is also the substrate for the polyamine metabolism, a major pathway used for cell growth and differentiation $(14,15)$. In the polyamine metabolism, arginine is converted by arginases into urea and ornithine that is further decarboxylated by ornithine decarboxylase (ODC), the rate limiting enzyme of the polyamine metabolism. The polyamine metabolism has been shown to play a crucial role in myocardial adaptation and transition to heart failure in the chronic pressure overloaded heart (16). Furthermore, the induction of the polyamine metabolism is linked to the activation of the RAS (17). An activation of arginase-1 in post-ischaemic hearts seems to indicate an activation of the polyamine metabolism (18).

The current study tested the hypothesis that events related to ischaemia/reperfusion may trigger a shift of the arginine metabolism from NO formation to polyamine metabolism and was aimed to identify potential mechanisms that trigger this event. A likely candidate may be a rapid release of local TNF- $\alpha$ by ischaemia-dependent activation of the sheddase" TNF- $\alpha$ converting enzyme" (TACE, also known as ADAM17) because TNF- $\alpha$ has been shown to induce the expression of arginase- 1 and an activation of arginase-1 was shown in post-ischaemic rat hearts (19-22). If this hypothesis holds, inhibition of TNF- $a$ release during ischaemia should avoid an activation of the arginine/polyamine pathway. In order to address these questions we performed in vitro experiments on isolated perfused rat hearts that were exposed to global no-flow ischaemia and reperfusion and added TAPI, an inhibitor of TNF- $\alpha$ release, during ischaemia. However, as pre-ischaemic TACE inhibition cannot be used in clinical practice we tested next, whether captopril can restore the pre-ischaemic balance between NO and polyamine metabolism by induction of the NO pathway.

In order to test this hypothesis captopril, an ACE inhibitor, was added to the perfusate of saline perfused rat hearts 30 minutes (min) after the onset of reperfusion. It has previously been shown that administration of sulfhydryl group containing ACE inhibitors can reduce infarct size if administered prior to ischaemia (i.e. [23]). Once again, as pre-ischaemic administration of therapeutics is not practicable in ischaemia/reperfusion in the clinical setting, captopril was given during late reperfusion in the subsequent experiments. Hearts were reperfused for 2 hours (h) and molecular markers of remodelling as well as the functional recovery were determined. Finally, we tested the effect of arginase inhibition during the onset of reperfusion. Unlike the above mentioned effects on transcription, a shift in arginine / eNOS activity may also be caused by uncoupling of NOS. In this case, inhibition of arginase should directly normalize the relationship between both arginine consumers.

\section{Material and methods}

\section{Animal models and animal handling}

The investigation conforms to the directive 2010/63/EU of the European Parliament. Use of animals was registered at the JustusLiebig-University (registration-no.: 417-M). The experimental protocols were approved by the ethics committee for animal experimentation of the local authorities in Giessen, Germany and Szeged, Hungary.

Myocardial infarction and reperfusion was performed in the closed-chest model. To achieve this, rats were anaesthetised by inhalation of isoflurane (induction: $5 \%$, maintenance: $2-3 \%$ ), intubated and placed on a respirator during surgery to maintain ventilation. Before surgery, $0.03 \mathrm{mg} / \mathrm{kg}$ nalbuphin (Nalbuphin Orpha, AOP Orpha Pharmaceuticals, Vienna, Austria) was injected (i.p.). The adequacy of anaesthesia was monitored by electrocardiography and pulse rate. A suture was placed around the left anterior descending coronary artery and remained subcutaneously (24). At $2 \mathrm{~h}$ after the wound closing, $0.03 \mathrm{mg} / \mathrm{kg}$ nalbuphin was repeated to alleviate postoperative pain. Seven days later, rats were anaesthetised as before and the suture was mobilised, and the LAD was occluded for $30 \mathrm{~min}$. The occlusion was monitored by electrocardiography (ST elevation). Thereafter, the occluder was opened again and the suture was cut and the skin was closed in one layer. Sham rats received the same protocol but the occluder was not mobilised after seven days. Rats that received captopril after ischaemia/reperfusion received the ACE inhibitor captopril (300 mg/l) in tap water (accumulating to approximately $30 \mathrm{mg} / \mathrm{kg}^{\star}$ day).

\section{Ex vivo analysis of cardiac function}

In order to analyse the cardiac function ex vivo, rats were anaesthetised again by isoflurane and killed by cervical dislocation. Thereafter hearts were rapidly excised and the aorta was cannulated for retrograde perfusion with a 16-gauge needle connected to a Langendorff perfusion system. Left ventricular function was determined by insertion of a water filled balloon into the left ventricle as described before (25). Hearts were paced during measurements.

\section{In vivo analysis of cardiac function}

Transthoracic echocardiography was performed as described previously (26) under isoflurane anaesthesia (1.5\%) at 120 days after ischaemia/reperfusion. Briefly, two-dimensional and M-mode echocardiographic examinations were performed in accordance with the criteria of the American Society of Echocardiography with a Vivid 7 Dimension ultrasound system (General Electric Medical Systems) using a phased array 5.5-12 MHz transducer (10S probe). Data of three consecutive heart cycles were analysed (EchoPac Dimension software; General Electric Medical Systems) by an experienced investigator in a blinded manner. The mean values of three measurements were calculated and used for statistical evaluation. Functional parameters including left ventricular 
ejection fraction (EF) and fractional shortening (FS) were calculated on four-chamber view images.

\section{qRT-PCR}

After removing the hearts from the Langendorff apparatus, the left ventricular tissue was carefully isolated and quickly frozen in fluid nitrogen. Tissue samples were prepared to analyse the steady-state mRNA levels of proteins of interest according to the previously described method (25). Briefly, total RNA was isolated from the left ventricles using peqGoldTriFast (peqlab, Biotechnology $\mathrm{GmbH}$, Erlangen, Germany) according to the manufacturer's protocol. To remove genomic DNA contamination, isolated RNA samples were treated with $1 \mathrm{U}$ DNase per mg RNA (Invitrogen, Karlsruhe, Germany) for $15 \mathrm{~min}$ at $37^{\circ} \mathrm{C}$. On $\mu \mathrm{g}$ of total RNA was used in $10 \mu \mathrm{l}$ reaction to synthesise cDNA using superscript RNaseH reverse

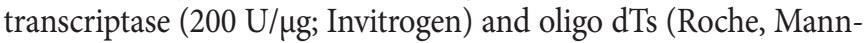
heim, Germany) as primers. Reverse transcriptase reactions were performed for $60 \mathrm{~min}$ at $37^{\circ} \mathrm{C}$. Real-time PCR was performed using the Icycler IQ detection system (Bio-Rad, Munich, Germany) in combination with IQ SYBR Green real-time supermix (Bio-Rad). A complete list of all primers used in this study is given in Table 1. Data are normalised to hypoxanthine phosphoribosyltransferase (HPRT) expression that was used as a housekeeping gene in this study. Preliminary experiments with $\beta_{2}$ microglobulin, which was alternatively considered as housekeeping gene, revealed similar results but higher variability. The relative change in expression was quantified by the $\Delta \Delta \mathrm{CT}$ method (27).

\section{Western blots}

Tissue samples from hearts stored at $-80^{\circ} \mathrm{C}$ were used as described previously and prepared for standard SDS gel electrophoresis. Protein was extracted as described before (25). The lysis buffer contained (mmol/l): Tris- $\mathrm{HCl}$ (pH 7.5) 20, $\mathrm{NaCl} 150$, EGTA 1, EDTA 2 , Triton ( $1 \% \mathrm{v} / \mathrm{v})$, sodium pyrophosphate (2.5), $\beta$-glycerophosphate $1, \mathrm{Na}_{3} \mathrm{VO}_{4}$, leupeptin $(1 \mu \mathrm{g} / \mathrm{ml})$ (Cell Signalling Technology, Leiden, Netherland). The homogenate was centrifuged at $1,000 \mathrm{~g}$ at $4^{\circ} \mathrm{C}$ for $10 \mathrm{~min}$ and the supernatant was used for protein detection by Western blotting. Supernatants were treated with Laemmli buffer (reducing conditions with $\beta$-mercaptoethanol; non-reducing conditions without $\beta$-mercaptoethanol). Samples were subsequently heated for $5 \mathrm{~min}$ at $95^{\circ} \mathrm{C}$ (reducing conditions) or incubated for $30 \mathrm{~min}$ at room temperature (non-reducing conditions). Protein samples were loaded on NuPAGE Bis-Tris Precast gels (10\%; Life Technology, Darmstadt, Germany) and subsequently transferred onto nitrocellulose membranes. Primary antibodies directed against eNOS , ODC, and cardiac $\alpha$-actin (loading control) were used as described before (27). Tropomyosin blots were incubated with an anti-tropomyosin antibody purchased from Sigma-Aldrich (Taufkirchen, Germany; product T9283). The antibody was previously used to establish tropomyosin disulphide cross-bridging (29).

\section{Measurement of superoxide}

To perform DHE staining, cryosections of left ventricles were incubated with DHE (dissolved in $1 \mathrm{X} \mathrm{PBS}$ ) for $10 \mathrm{~min}$ at $37^{\circ} \mathrm{C}$ in a light-protected humidity chamber, then fixed with Dako Fluorescent Mounting Medium (Dako, Glostrup, Denmark). Slides were then imaged by confocal microscopy (LSM 510 META; Carl Zeiss, Jena, Germany) using an excitation wavelength of $488 \mathrm{~nm}$; emission was recorded at $540 \mathrm{~nm}$. Assessment was performed by digital image analysis using Leica Confocal Software Lite Version (LCS Lite). The mean fluorescence intensity of $n=8$ per group was used to quantify the extent of superoxide.

\section{Quantification of TNF- $\alpha$ concentrations}

Langendorff heart-perfusates were collected prior to ischaemia and one minute after reperfusion and filtered through $0.2 \mu \mathrm{m}$ filter to remove any residual debris. To quantify the TNF- $\alpha$ production, an enzyme-linked immunosorbent assay (ELISA) (Quantikine, R\&D Systems, Minneapolis, MN, USA) was used. Absorbance values for individual reactions were determined using VersaMax ${ }^{\mathrm{Tm}}$ Microplate Reader with SoftmaxPro 3.0 data processing software.

\section{Statistics}

The results are expressed as means \pm S.E.M. or box plots as indicated in the legend to the figures. Statistical comparisons were performed by two-side ANOVA and Student-Newman-Keuls posthoc analysis. Levene test was used to check the normal distribution

\begin{tabular}{l|l|l}
\hline & Forward & Reverse \\
\hline HPRT & CCA GCG TCG TGA TTA GTG AT & CAA GTC TTT CAG TCC TGT CC \\
\hline eNOS & AGC CCG GGA CTT CAT CAA TCA & GCC CCA AAC ACC AGC TCA CTC TC \\
\hline ODC & GAA GAT GAG TCA AAC GAG CA & AGT AGA TGT TTG GCC TCT GG \\
\hline Arginase-1 & GGA AGC ATC TCT GGC CAC GCC & CAC CGG TTG CCC GTG CAG \\
\hline Arginase-2 & TGA GGA GCA GCG TCT CCC GT & GCT TCT CGG ATG GCG GCT GG \\
\hline ANP & ATG GGC TCC TCC TCC ATC AC & TCT TCG GTA CCG GAA GCT \\
\hline SERCA2a & CGA GTT GAA CCT TCC CAC AA & AGG AGA TGA GGT AGC GGA TGA A \\
\hline Bax & ACT AAA GTG CCC GAG CTG ATC CAC & TGT CTG CCA TGT GGG G \\
\hline
\end{tabular}

Table 1: RNA primer sequences. 
Figure 1: Left ventricular mRNA expression of arginase 1, eNOS, ODC and the ODC-toeNOS ratio in hearts exposed to $45 \mathrm{~min}$ ischaemia and $120 \mathrm{~min}$ reperfusion. HPRT was used to normalise the data. Data are shown as box plots and individual data points outside the $5 \%$ and $95 \%$ are indicated separately. The dashed line indicates basal expression of control hearts $(\mathrm{Nx})$ not undergoing $45 \mathrm{~min}$ of ischaemia and reperfusion (I/R). TAPI $(1 \mu \mathrm{mol} / \mathrm{l})$ was used to inhibit the activity of TACE. * ${ }^{*} p<0.05$ vs Nx ( $n=6-8$ hearts).
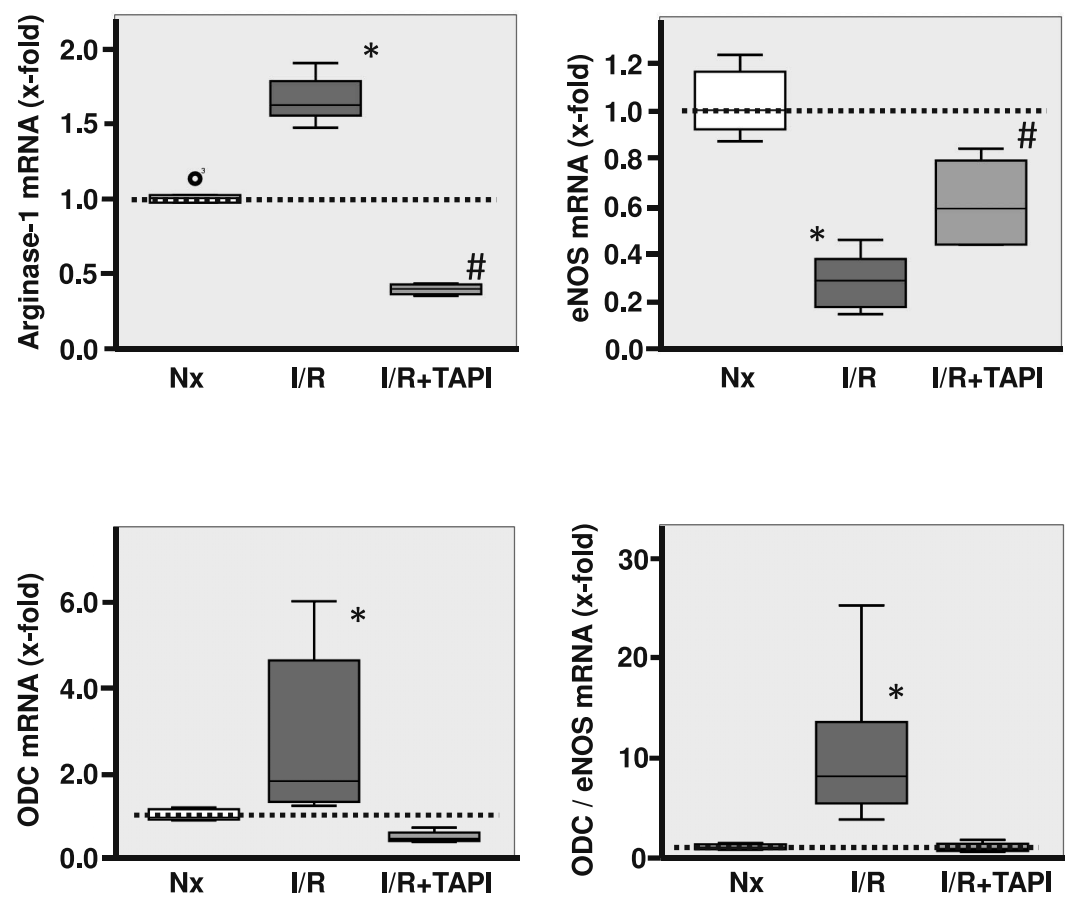

of the samples. A p-value of 0.05 was considered as statistical significant.

\section{Results}

\section{Influence of TAPI administration during ischaemia on the functional recovery of post-ischaemic hearts and the expression of genes linked to arginine metabolism}

In the first set of experiments we tested the hypothesis that TNF- $\alpha$ released during ischaemia may trigger a molecular switch of arginine metabolism during reperfusion by an activation of the sheddase "TNF- $\alpha$ converting enzyme" (TACE). In order to address this question we administered the TACE inhibitor TAPI $(1 \mu \mathrm{M})$ to hearts prior to the onset of ischaemia. $\$$ Figure 1 describes the subsequent changes in mRNA expression of arginase-1, eNOS, ornithine decarboxylase (ODC), and the ratio between eNOS and ODC. Ischaemia/reperfusion increased arginase-1 mRNA expression, whereas a decreased mRNA expression of eNOS and increased mRNA expression of ODC shifted the eNOS/ODC ratio into the direction of polyamine metabolism. Noteworthy, all these changes were completely attenuated if TAPI was administered prior to reperfusion. However, these molecular changes on mRNA expression had no effects on the functional heart recovery within 2 h. At $2 \mathrm{~h}$ after reperfusion, hearts developed a mean left ventricular pressure of $59.4 \pm 8.9 \mathrm{mmHg}$ in control hearts and $63.4 \pm 5.8$ $\mathrm{mmHg}$ in hearts treated with TAPI $(\mathrm{p}=0.717, \mathrm{n}=6)$. Pre-ischaemic values had been $95.1 \pm 2.6 \mathrm{mmHg}$ and $96.2 \pm 6.3 \mathrm{mmHg}$, respectively. As expected, TAPI significantly attenuated the release of TNF- $\alpha$ into the perfusate. This amount of TNF- $\alpha$ was $13.0 \pm 4.3$ $\mathrm{pg} / \mathrm{ml}$ in the perfusate of normoxic control hearts and increased to $52.5 \pm 10.9 \mathrm{pg} / \mathrm{ml}$ ( $\mathrm{p}<0.05 ; \mathrm{n}=6$ hearts) in the perfusate of post-ischaemic hearts. TAPI reduced this increase to $19.6 \pm 9.8 \mathrm{pg} / \mathrm{ml}$. Collectively these data show that ischaemia/reperfusion shifts the arginine pathway into the direction of polyamine metabolism and identify TNF- $\alpha$ as a causative factor in this process. Whether this process can be reversed by inhibition of the local RAS was tested next.

\section{Functional recovery of rat hearts after ischaemia/- reperfusion and influence of captopril}

In order to address the question whether ACE inhibition can antagonise TNF- $\alpha$-driven molecular changes of ischaemia/reperfusion, the ACE inhibitor captopril was added $30 \mathrm{~min}$ after the onset of reperfusion. Again, there were no functional differences between the treatment group and the captopril group. Pre-ischaemic values of left ventricular developed pressure (LVDP) were $115.3 \pm$ $6.1 \mathrm{mmHg}$ and $104.9 \pm 6.6 \mathrm{mmHg}$. At $30 \mathrm{~min}$ after the onset of reperfusion and before starting captopril administration LVDP values were $85.9 \pm 10.6 \mathrm{mmHg}$ and $85.4 \pm 5.3 \mathrm{mmHg}$, respectively. Finally, LVDP values amounted to $50.5 \pm 5.2 \mathrm{mmHg}$ and $48.0 \pm 5.7$ $\mathrm{mmHg}$, respectively, $2 \mathrm{~h}$ after the start of reperfusion. Thus, captopril did not affect the functional recovery of hearts when administered $30 \mathrm{~min}$ after reperfusion and present for further $90 \mathrm{~min}$. 


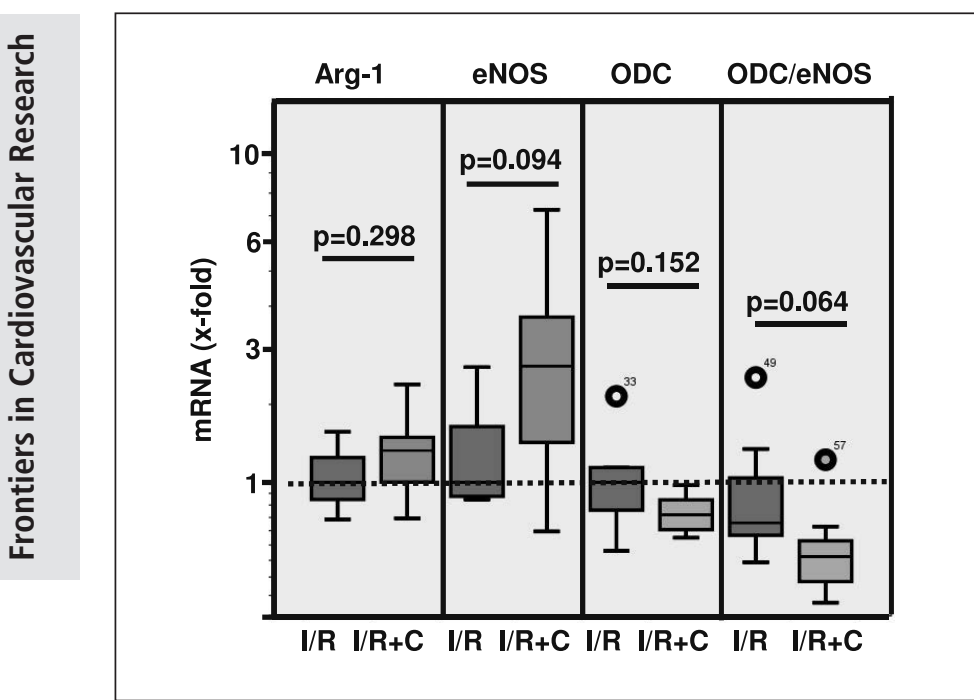

Figure 2: Left ventricular mRNA expression of arginase 1, eNOS, ODC and the ODC-to-eNOS ratio in hearts exposed to $45 \mathrm{~min}$ ischaemia and 120 min reperfusion. HPRT was used to normalise the data. Data are shown as box plots and individual data points outside the $5 \%$ and $95 \%$ are indicated separately. The dashed line indicates basal expression of control hearts (I/R) not receiving captopril $(100 \mu \mathrm{mol} / \mathrm{l})$ starting $30 \mathrm{~min}$ after the onset of reperfusion. Exact $\mathrm{p}$-values are given (each $\mathrm{n}=8$ ).

In contrast to the lack of effect on functional recovery, a clear trend to higher expression of eNOS following captopril was found. Moreover, the mRNA expression of arginase-1 and ODC was lower than in the non-treatment group ( Figure 2). Collectively, these data show that inhibition of the local ACE system potentially

Table 2: Effect of captopril on cardiac function ex vivo 24 and 72 hours after ischemia/reperfusion.

\begin{tabular}{l|l|l}
\hline & LVDP $(\mathrm{mmHg})$ & LVDP (\%) \\
\hline Day 1 (24 h) & & \\
\hline Sham & $158 \pm 7$ & $100 \pm 4$ \\
\hline I/R & $123 \pm 7$ & $78 \pm 4$ \\
\hline$\Delta$ & $-35^{*}$ & $-22^{*}$ \\
\hline Sham / Capto & $186 \pm 11$ & $100 \pm 6$ \\
\hline I/R / Capto & $165 \pm 5$ & $89 \pm 2$ \\
\hline$\Delta$ & -21 & -11 \\
\hline Day 3 (72 h) & & \\
\hline Sham & $155 \pm 8$ & $100 \pm 5$ \\
\hline I/R & $132 \pm 10$ & $85 \pm 7$ \\
\hline$\Delta$ & -23 & -15 \\
\hline Sham / Capto & $168 \pm 8$ & $100 \pm 5$ \\
\hline I/R / Capto & $153 \pm 8$ & $91 \pm 5$ \\
\hline$\Delta$ & -15 & -9 \\
\hline Data are means S.E.M. from n=8 hearts each. & \\
\hline * $\mathrm{p}<0.05$ vs. Sham. & & \\
\hline
\end{tabular}

attenuates the shift of the arginine metabolism by up-regulation of eNOS. The data in the in vitro model show significant effects on the mRNA expression of enzymes involved in the arginine metabolism. However, these changes seem not to be functionally relevant within minutes because these changes have to be translated into proteins first. Whether this shift in arginine metabolism modifies the outcome at later time points was investigated next in an appropriate in vivo model.

\section{Functional recovery of rats after in vivo ischaemia/ reperfusion and effect of administration of captopril}

In order to address the functional relevance of eNOS up-regulation by captopril, rats underwent ischaemia and reperfusion in the so-called closed chest model. Rats received either captopril with their tap water directly after recovery from the surgery for one or three days (treatment group) or no drug (non-treatment group). Control rats underwent sham surgery without ligation of the left anterior descending artery. Cardiac function was analysed ex vivo in saline perfused rat hearts. Post-ischaemic rats developed a loss of cardiac function at day 1 after reperfusion that was less pronounced at day 3 after reperfusion ( Table 2). Of note, this early loss of function was largely attenuated in the captopril group. When the mRNA expression of eNOS, arginase-1 and ODC was analysed in post-ischaemic hearts from rats of the control group in comparison to the captopril group, they displayed significant differences. As indicated in $>$ Figure 3 , the administration of the ACE inhibitor induced down-regulation of the mRNA expression of ODC and normalised the expression of eNOS. On the protein level, ODC mRNA expression was higher in post-ischaemic nontreated hearts, and the inhibitor attenuated the induction of ODC. It also normalised the expression of eNOS and the ODC-to-eNOS ratio.

The differences between treated and non-treated rats in left ventricular function were lower at day 3 ( $\$$ Table 2) and at that time-point the molecular adaptations were also smaller compared to day 1 but all changes displayed a similar trend than at day 1 ( Figure 4). In addition, the induction of arginase-1 by ischae$\mathrm{mia} /$ reperfusion remained significant at day 1 after reperfusion but was again normalised by captopril administration ( Figure 5). This effect of ischaemia/reperfusion on arginase expression was lost within the subsequent days ( $\$$ Figure 5). In summary, captopril shifted the arginine metabolism into the direction of NO metabolism. This effect was induced at a very early time point as seen in the saline perfused rat hearts (see above, although not functionally significant at this time point), strongest at day 1 after reperfusion, but already beginning to be lost within the next days in the presence of captopril.

Mechanistically the question occurred whether the attenuation of the shift in arginine metabolism may cause decreased oxidative stress. As expected, ischaemia/reperfusion caused an increase in superoxide formation as indicated by increased DHE staining (Suppl. Figure 1a, b, available online at www.thrombosis-online. com). As recently demonstrated, oxidative modification of tropomyosin leads to myocardial dysfunction by TNF- $\alpha$-dependent in- 
Figure 3: Protein and mRNA expression of eNOS and ODC in rat hearts from rats undergoing $30 \mathrm{~min}$ of ischaemia and $24 \mathrm{~h}$ of reperfusion in vivo. Where indicated, rats received captopril by tapwater after surgery. Data are shown as box plots and individual data points outside the $5 \%$ and $95 \%$ are indicated separately. The dashed line indicates basal expression of control hearts (Sham) not receiving captopril. Exact $\mathrm{p}$-values are given (each $n=8)$.
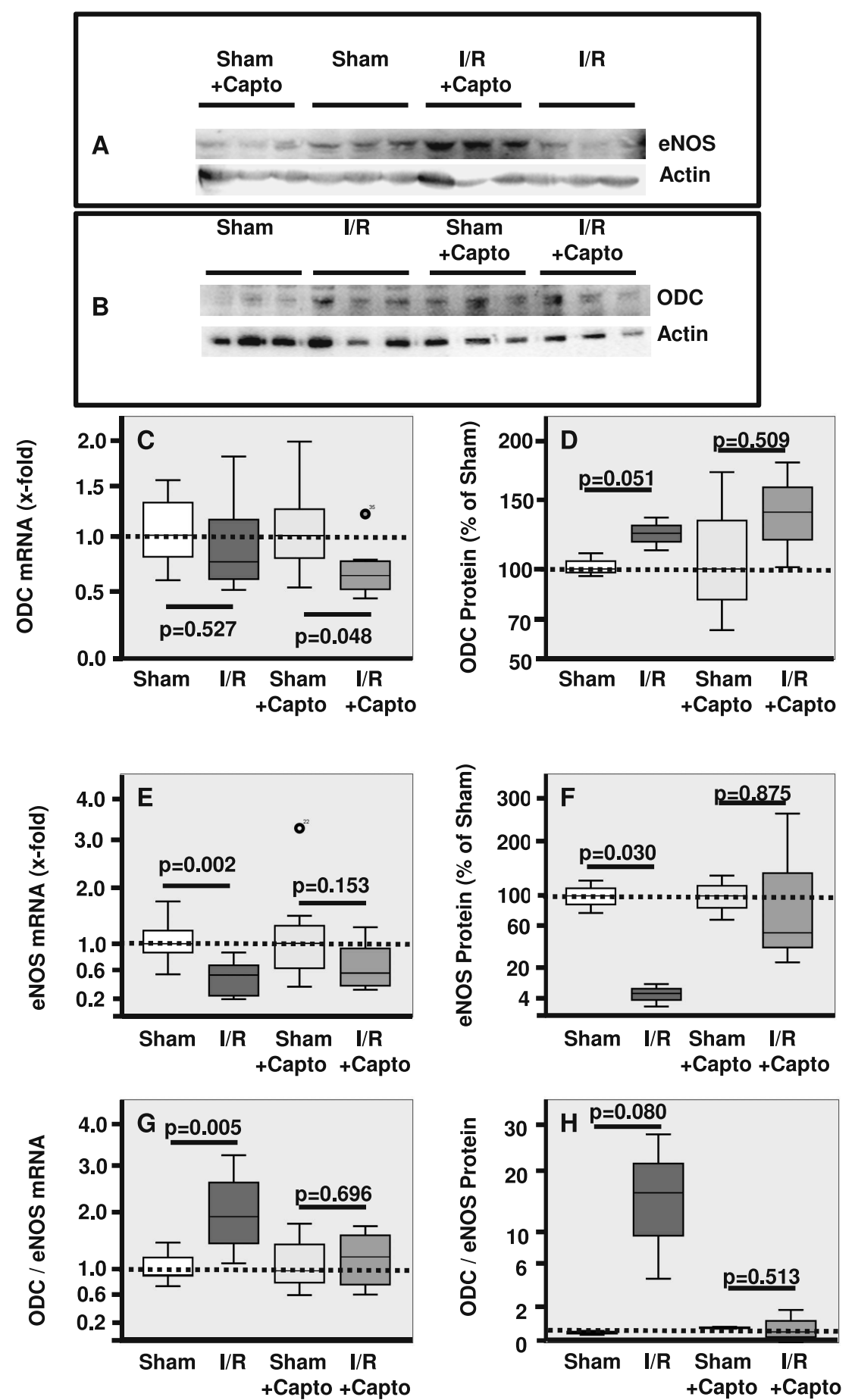

duced oxidative stress. As shown in Suppl. Figure 1c (available online at www.thrombosis-online.com), under non-reducing conditions the anti-tropomyosin antibody detected a band at approximately $45 \mathrm{kDa}$ corresponding to a band detected under reducing conditions as well, and an additional band at a much higher molecular weight appeared (approximately at $80 \mathrm{kDa}$ ), which most likely was caused by disulphide cross-bridges (DCB; [29]). Ischae$\mathrm{mia} /$ reperfusion resulted in a higher degree of DCB amounts and this was no longer significantly enhanced if captopril was administered during the first day (Suppl. Figure 1c, available online at www.thrombosis-online.com). 


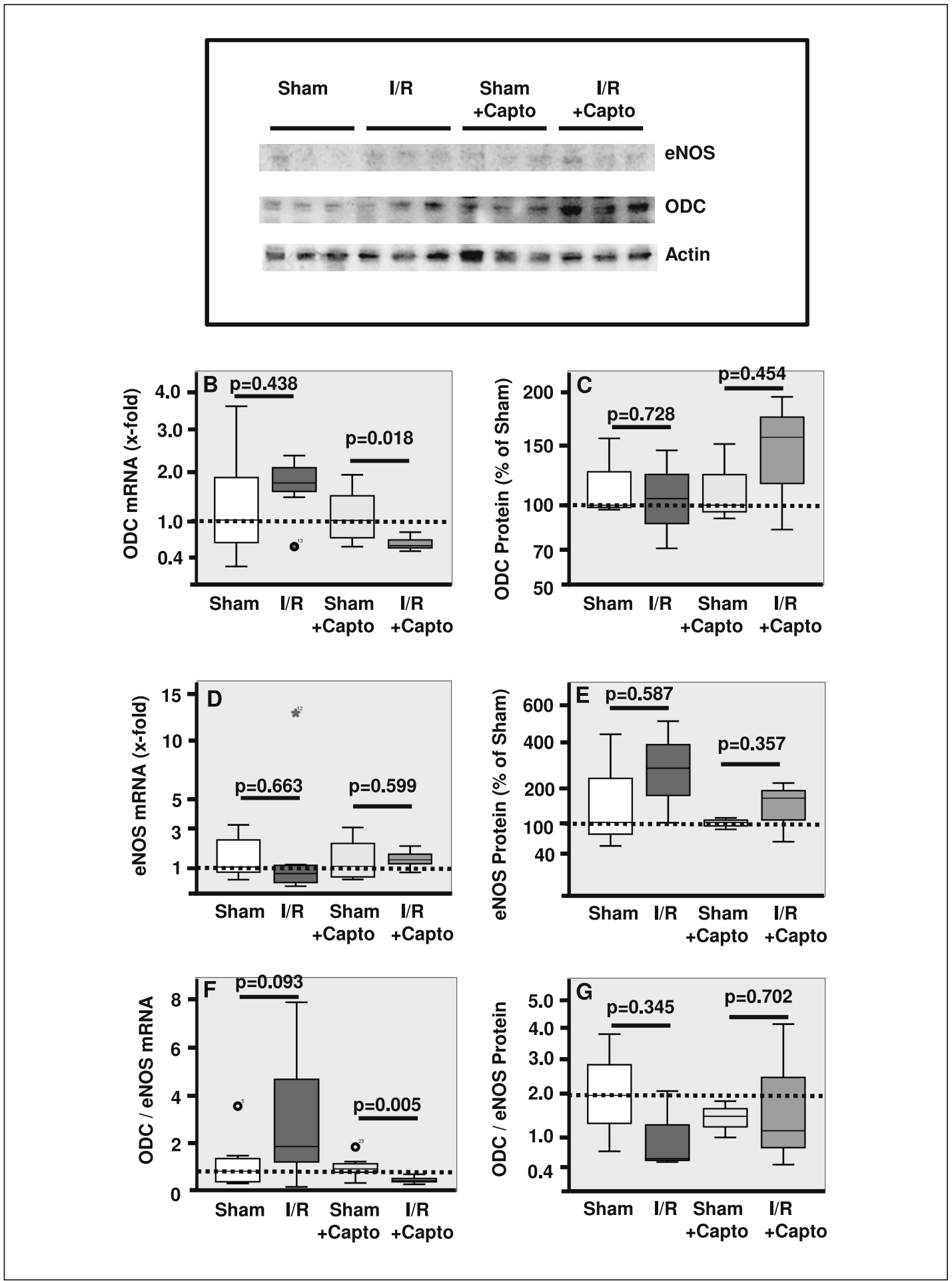

Figure 4: Protein and mRNA expression of eNOS and ODC in rat hearts from rats undergoing $30 \mathrm{~min}$ of ischaemia and $72 \mathrm{~h}$ of reperfusion in vivo. Where indicated, rats received captopril by tapwater after surgery. Data are shown as box plots and individual data points outside the $5 \%$ and $95 \%$ are indicated separately. The dashed line indicates basal expression of control hearts (Sham) not receiving captopril. Exact $\mathrm{p}$-values are given (each $\mathrm{n}=8$ ).

\section{Functional recovery of rats after in vitro ischaemia/ reperfusion and effect of administration of Nor-NOHA}

The results shown above have clearly shown the impact of arginase/ODC and eNOS expression on post-ischaemic recovery within $24 \mathrm{~h}$. Without changes in expression, a similar shift may occur at the onset of reperfusion due to eNOS uncoupling (see Introduction). Whether an increased activity of arginase relative to eNOS can contribute to oxidative stress, namely superoxide formation, and directly depress functional recovery was investigated next. To address this question, arginase activity was inhibited by Nor-NOHA, a specific inhibitor of arginase during rep- 
Figure 5: Left ventricular mRNA expression in rat hearts from rats undergoing $30 \mathrm{~min}$ of ischaemia and $24 \mathrm{~h}$ $(A, B)$ or $72 \mathrm{~h}(C, D)$ of reperfusion in vivo. Data are shown as box plots and individual data points outside the $5 \%$ and $95 \%$ are indicated separately. The dashed line indicates basal expression of control hearts (Sham) not receiving captopril. Exact p-values are given (each $n=8$ ).
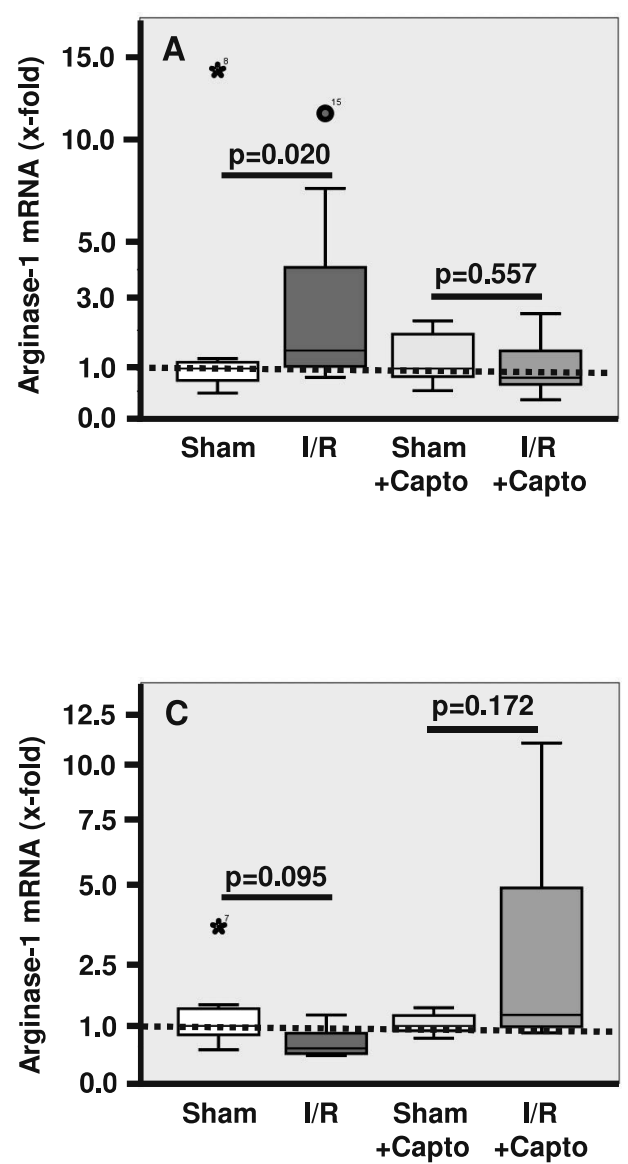
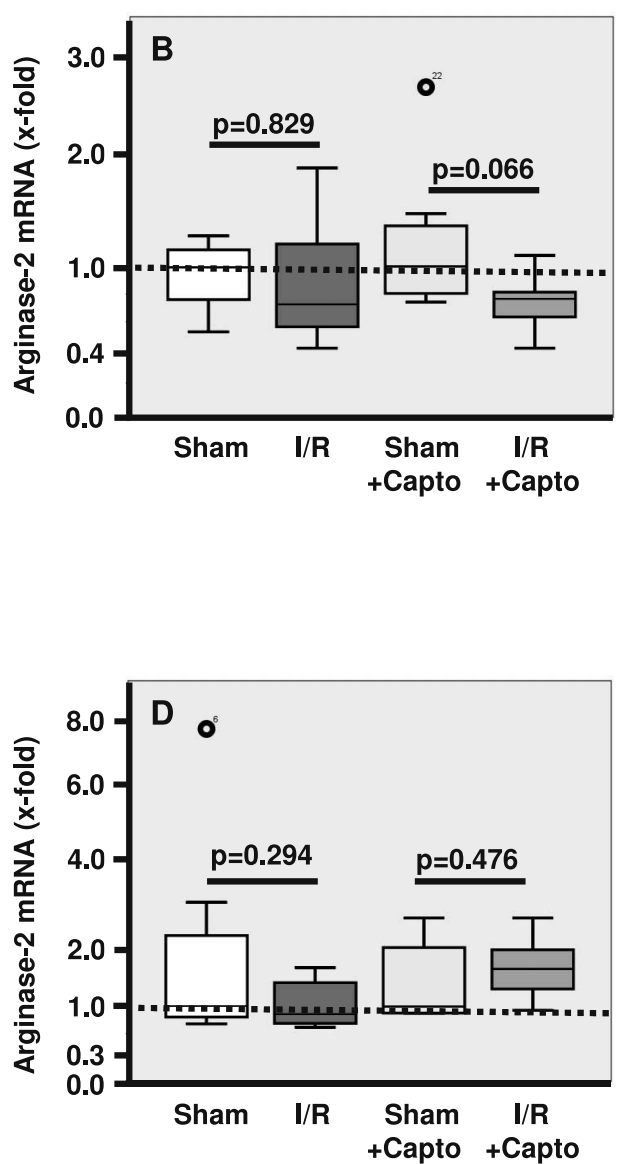

erfusion. As expected, ischaemia/reperfusion caused an increase in superoxide formartion as indicated by increased DHE staining ( Figure 6A, B). However, inhibition of arginase activity during reperfusion caused a significant reduction of oxidative stress and improved the functional recovery of these hearts ( $\triangleright$ Figure $6 \mathrm{~A}, \mathrm{~B}$ ). This increased oxidative stress went along with a clear shift in tropomyosin oxidation ( $\$$ Figure $6 \mathrm{C}$ ). In addition, the functional recovery (120 min reperfusion) was significantly improved in hearts that received Nor-NOHA at the onset of reperfusion [LVDP: $57.4 \pm 9.2 \mathrm{mmHg}$ (Nor-NOHA; $\mathrm{n}=8$ ) vs $44.1 \pm 9.2 \mathrm{mmHg}$ (Control; $\mathrm{n}=11$ ); $\mathrm{p}<0.05]$. Pre-ischaemic values were $116.4 \pm 20.5$ and $121.1 \pm 20.4 \mathrm{mmHg}$, respectively. These data suggest that the shift in arginine metabolism is associated with changes in the oxidative stress affecting directly the cardiac function. Collectively the data suggest that activation of the local TNF-a system convert the acute activation of arginase into sustained arginase activation by transcriptional activation and that this process can be attenuated by induction of the counter-regulatory eNOS by ACE inhibition.

\section{Long-term effect of the captopril-driven attenuation of the shift in arginine metabolism in the early phase of reperfusion}

Finally, we examined whether this early protection by captopril has any long-term consequences for the post-ischaemic recovery. At day 120, no differences in the mRNA expression of molecules linked to arginine metabolisms (eNOS, arginase-1, ODC) were found between both groups of ischaemia/reperfusion (Suppl. Figure 2, available online at www.thrombosis-online.com). Surprisingly, although captopril treatment had a significant better functional recovery of the heart during the first days after reperfusion in vivo, this did not end up in any functional improvements after four months. This was indicated in vivo by a similar reduction of EF compared to sham rats in treated and non-treated rats, and confirmed in vitro by a lower LVDP in saline reperfused rat hearts. However, although no functional effect was found in these rats three molecular markers of cardiac hypertrophy and remodelling showed significant difference in their mRNA expression between captopril-treated and non-treated rats. These were ANP, a marker of cardiac hypertrophy that was elevated in the captopril group, SERCA2a, a calcium transporter that improves cardiac function by 
A

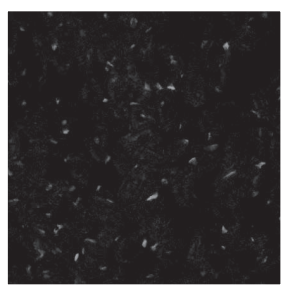

Control

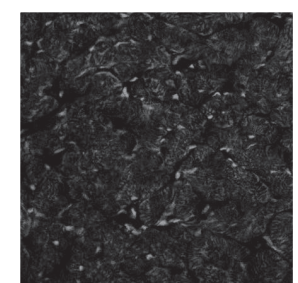

I/R

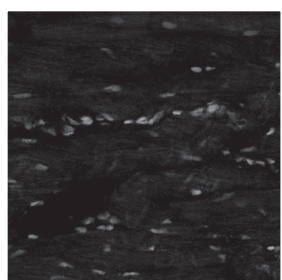

I/R + Nor-NOHA

B

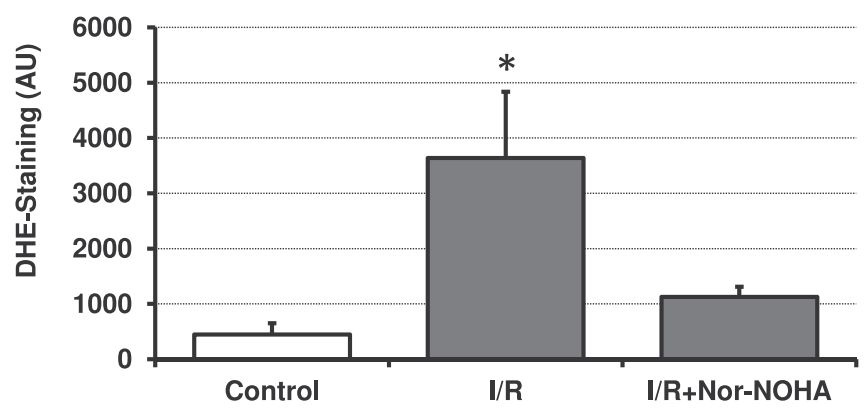

C

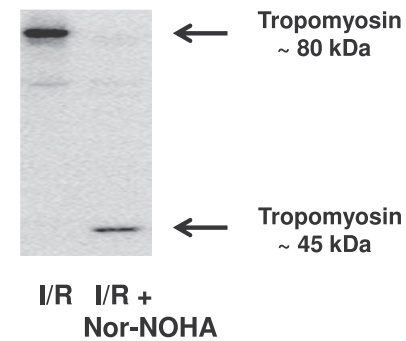

Figure 6: Superoxide formation in post-ischaemic hearts and its modification by arginase inhibition. Data are shown for control hearts (normoxic perfusion), post-ischaemic hearts (30 min after reperfusion; I/R), and hearts receiving the arginase inhibitor Nor-NOHA $(100$ $\mu \mathrm{M})$ during reperfusion. A) Representative tissue slices with superoxide staining by DHE in green. B) Quantitative analysis of DHE staining for the three groups (each $n=8$ ). ${ }^{*}, \mathrm{p}<0.000$ vs control and Nor-NOHA group. C) Representative immunoblots of tropomyosin under non-reducing conditions. acceleration of calcium refilling of the sarcoplasmatic reticulum that was also higher expressed in the captopril group, and bax, a pro-apoptotic factor, which was less expressed in these rats ( $>$ Figure 7). These changes suggest a slightly different type of compensatory hypertrophy in captopril-treated rats.

\section{Discussion}

This study demonstrated that at the onset of reperfusion and several hours after reperfusion a shift within the arginine metabolism occurs that favours arginine consumption into the polyamine metabolism and reduces NO formation. Initially, this shift is triggered by eNOS uncoupling. Inhibition of arginase normalises the ratio between the activities of both arginine consumers and thereby reduces oxidative stress. This shift in arginine metabolism is maintained at later time points by an induction of arginase- 1 and ODC mRNA and protein expression and a down-regulation of eNOS mRNA expression. As a potential mechanism that triggers this transcriptional activation, we identified ischaemia/reperfusion-dependent activation of TACE. As a new finding of this study we show for the first time that this shift in arginine metabolism can be normalised by inhibition of the cardiac RAS. This inhibition attenuates the down-regulation of eNOS. Under these conditions, cardiac function was maintained at day 1 after reperfusion. Mechanistically, it is suggested that $\mathrm{NO}$ as a radical acceptor binds ROS that would otherwise oxidise cysteine residues at tropomyosin and thereby reduce cardiac function. In this study we show that inhibition of arginase during reperfusion indeed reduces superoxide formation and improves the early functional recovery and that interference with arginase up-regulation and eNOS down-regulation improves the situation within hours. Despite these promising results the long-term cardioprotective effect is low in terms of functional aspects but is associated with moderate molecular adaptations on the mRNA expression level. A schematic overview about the proposed mechanism is given in $>$ Figure 8 .

It has previously been shown that ischaemia leads to an induction of arginase- 1 that converts arginine into ornithine so that arginine is no longer available as a substrate of eNOS. At least in the rat arginase-1 is the main isoform (18). Our study confirmed these findings and documented that within minutes after reperfusion argininase-1 mRNA expression increases in isolated saline perfused 

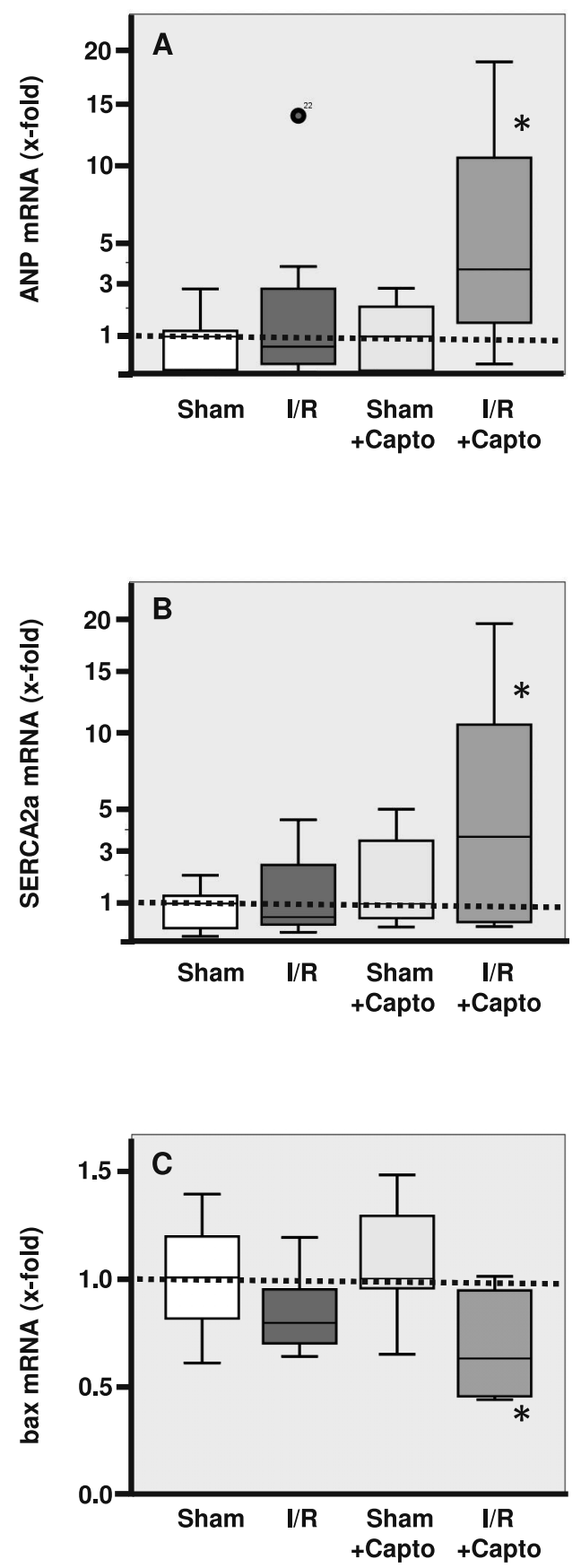

Figure 7: Left ventricular mRNA expression of ANP, SERCA2a, and bax in hearts exposed to $30 \mathrm{~min}$ ischaemia and 120 days of reperfusion. HPRT was used to normalise the data. Data are shown as box plots and individual data points outside the $5 \%$ and $95 \%$ are indicated separately. The dashed line indicates basal expression of control hearts (Sham) not receiving captopril. ${ }^{*}, p<0.05$ vs Sham (each $n=8$ ).

rat hearts its protein expression is increased $24 \mathrm{~h}$ after ischaemia/ reperfusion in vivo. Furthermore, although it is unlikely that an induction of arginase expression leads to a functional active enzyme

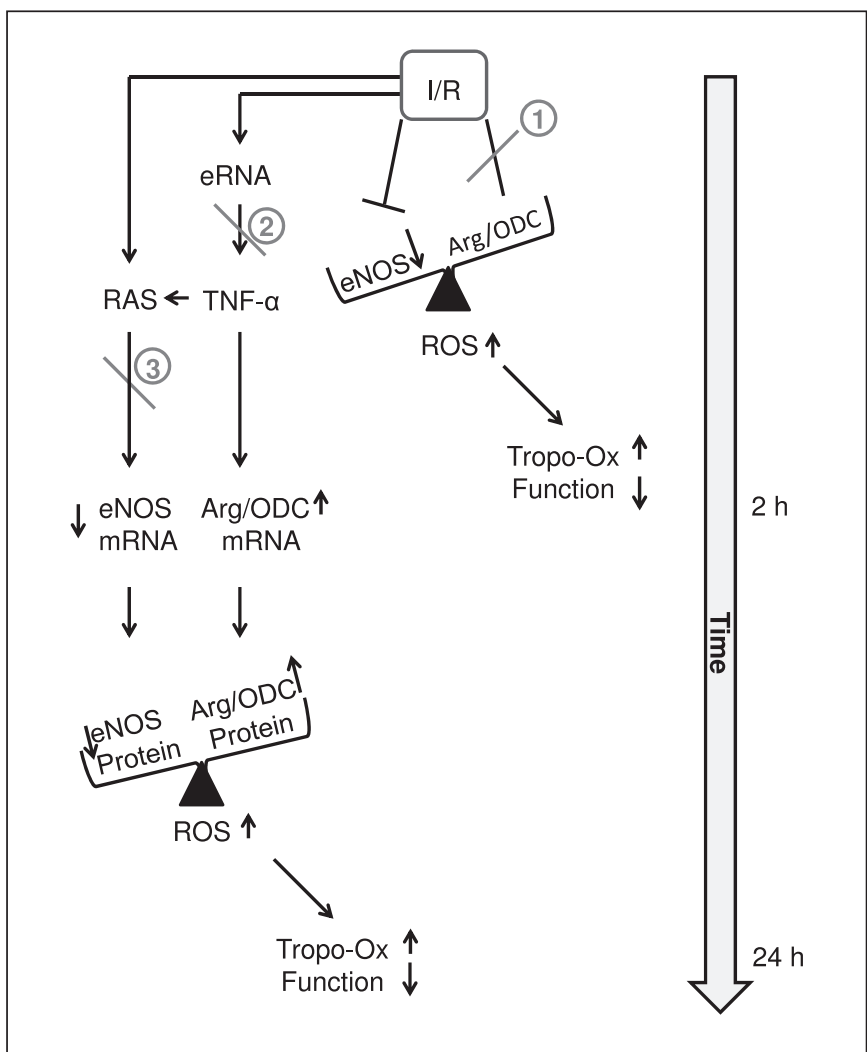

Figure 8: Summary figure - Ischaemia/Reperfusion (I/R) uncouples eNOS and thereby shifts the relationship between arginase / ODC and eNOS into the direction of arginase. This causes oxidative stress, oxidation of tropomyosin and loss of function. Inhibition of arginase (red labelled No. 1) normalised the relationship between arginase and eNOS. I/R also led to release of cell compounds due to loss of sarcolemmal integrity including RNA. Extracellular RNA (eRNA) activates TACE leading to liberation of bound TNF- $\alpha$. TNF- $\alpha$ induces the expression of arginase and ODC and activates the renin-angiotensin-system (RAS) that down-regulated the expression of eNOS. The subsequent unbalance between arginase / ODC and eNOS causes again oxidative stress, oxidises tropomoysin, and reduces function. Please note the time scale. Liberation of TNF- $\alpha$ can be attenuated by inhibition of TACE (red labelled 2), RAS can be attenuated by captopril (red labelled 3). Inhibition of arginase has acute effects, inhibition of TACE or RAS delayed effects.

within minutes our data show that we can attenuate the formation of oxidiative stress by inhibition of arginase. The most likely explanation for these acute effects are eNOS uncoupling at the time of reperfusion $(10,11)$. Our finding that arginase- 1 is strongly induced in vitro suggests that local mechanisms may trigger this increase. Arginase is known to be induced by TNF-a (22). In the context of ischaemia/reperfusion, local release of TNF- $\alpha$ triggered by TACE may induce this response. We hypothesised that TACE will be activated by ischaemic events and liberates TNF- $a$ because it is reasonable to assume that necrotic cell death during ischaemia will lead to the release of cellular components like RNA that subsequently may activate TACE (19). Indeed, an inhibition of TACE blocked reduced TNF- $a$ concentration in the perfusate and attenuated arginase-1 induction. 
Although induction of arginase-1 may already indicate a shift of the arginine metabolism into the direction of the polyamine metabolism, the rate limiting enzyme of the polyamine metabolism is not arginase-1 but ODC. Of note, based on the analysis of arginase knockout mice, arginase expression and activity seems not to affect polyamine metabolism directly at least in healthy mice underlining the importance of ODC expression in the induction of polyamine metabolism (30). Interestingly, in the setting of cardiac ischaemia/reperfusion arginase-1 and ODC were co-induced in vitro. In the in vivo model protein expression of ODC was higher $24 \mathrm{~h}$ after the onset of reperfusion, although only at a borderline level of significance $(p=0.051)$. Nevertheless, these data suggest that the induction of the polyamine metabolism in post-ischaemic hearts includes ODC.

From these experiments the question arises how TNF- $a$ may trigger these changes with an adverse outcome. It is well known that an inhibition of the local RAS in post-infarct models improves the post-ischaemic heart recovery at least in a certain time window (see Introduction). Can it be that TNF- $\alpha$ activates a local RAS that then triggers the shift into the polyamine metabolism? Evidence for this hypothesis comes from experiments with transgenic mice with a restricted overexpression of TNF- $\alpha$ in the heart. These mice displayed an activation of the cardiac-specific RAS (31). Combining both findings, TNF- $\alpha$ may indeed activate a local RAS that then triggers the shift in the polyamine metabolism. Inhibition of the local RAS 30 min after the onset of reperfusion in the in vitro system induced eNOS expression and increased the eNOS-toODC ratio on the level of mRNA expression. This was not accompanied by a rapid functional improvement at that time. However, one cannot expect such an effect within $90 \mathrm{~min}$ after treatment because this may be too early for changes on the protein expression level. ACE inhibition significantly preserved the function compared to non-treated rats in vivo, although treatment was started late after reperfusion. The timing of the start of captopril treatment was based on previous findings that earlier treatment is not successful and excludes any effect on infarct size that is established within minutes after reperfusion (3). Of note, this treatment regime led to a normalisation of eNOS expression and moderate effects on arginase-1 and ODC expression as well. These promising effects were lost during the following days even in the presence of the ACE inhibitor. Collectively our data show a significant contribution of local RAS to the arginine switch that has functional consequences during the first days after reperfusion.

So far the data indicated that the post-ischaemic shift of the arginine metabolism is initiated by ischaemia-dependent activation of TNF- $\alpha$, that then triggers an activation of the RAS. The final question to answer is, however, whether this leads to a long-term protection against the transition of the pre-ischaemic healthy heart into post-ischaemic heart failure. The closed chest model limits any inflammation linked to the experimental procedure and limits inflammation to the infarct healing-related aspects. This allowed to follow-up the animals for longer time periods up to four months. As expected, post-ischaemic rats developed a reduced cardiac function as shown by echocardiography. However, even rats that previously received the ACE inhibitor during the first seven days after reperfusion, in which wound healing and remodelling starts, still developed signs of heart failure. There was no functional difference between both groups after four months. Nevertheless, the left ventricular mRNA expression of ANP and SERCA2a were highest in these rats whereas the expression of the pro-apoptotic bax was lowest. Interestingly, we have previously shown that putrescine activates bax expression immediately after reperfusion, while inhibition of ODC activity reduced the bax expression (32). Collectively the data suggest that polyamines can affect bax mRNA expression and that ACE inhibition attenuates this effect. Any speculation about the contribution of altered bax mRNA expression on the rate of apoptosis, however, cannot be drawn from this study. Nevertheless, the data underline an effect of temporary ACE inhibition on post-infarct remodelling. The long-term functional consequences cannot be predicted at the present state and require further studies.

The aforementioned changes in cardiac expression of enzymes of the arginine metabolism in vivo are not due to changes in differences in infarct sizes as treatment stared at earliest $30 \mathrm{~min}$ after reperfusion, a time at which infarct size has already been established (see above). In the in vitro experiments performed here, infarct sizes can also not be impaired in the captopril experiments. In case of TACE and arginase inhibitors had to be given at earlier time points because their activity is critically involved at earlier time points. However, based on protein leakage from perfused rat hearts there was no difference in infarct sizes (data not shown). Collectively the data show that arginase metabolism can be modified independent of infarct sizes.

As mentioned above, timing of drug administration and type of linkage of ROS to tropomyosin oxidation suggest that the manipulation of arginine metabolism are direct consequences of ROS scavenging rather than consequences of cell death. The data are also consistent with the assumption that some of the cardiac depressive mechanisms at the early post-ischaemic period are transient effects as those shown in this study.

In summary, the current study describes new mechanisms how ischaemia/reperfusion leads to a shift in arginine metabolism during the early phase of reperfusion, how this can be attenuated and that this improves the early functional recovery. Importantly, the improved cardiac function observed during the initial phase after reperfusion indicates that this mechanism may significantly contribute to the early reduction of cardiac function after myocardial infarction and reperfusion. The study also shows that this mechanism is not part of the subsequent remodelling process leading to heart failure in post-ischaemic hearts and that a better initial recovery does not attenuate this process either. However, the early phase after reperfusion is a critical phase in general and any improvement of cardiac function at this time will probably reduce post-reperfusion complications.

\section{Acknowledgement}

We thank Nadine Woitasky and Peter Volk for excellent technical support. The data of this study are in part results of the thesis of Pia Weber. The work was supported in part by the German Research Council (DFG, Bonn, Germany) with the international 
Graduate School PROMISE (IRTG-1566, Giessen-Barcelona), the excellent Cluster „Cardiopulmonary System“ (ECCPS) strat-up grant to HACF, and a university hospital-Giessen-Marburg grant. PB and CC hold Janos Bolyai Research Scholarship of the Hungarian Acedemy of Science.

\section{Conflicts of interest}

None declared.

\section{References}

1. Goel K, Pinto DS, Gibson M. Association of time to reperfusion with left ventricular function and heart failure in patients with acute myocardial infarction treated with primary percutaneous coronary intervention: A systematic review. Am Heart J 2013; 165: 451-467.

2. Brodie BR, Stone GW, Cox DA, et al.. Impact of treatment delays on outcomes of primary percutaneous coronary intervention for acute myocardial infarction: Analysis from the CADILLAC trial. Am Heart J 2006; 151: 1231-1238.

3. Schoemaker RG, Debets JJM, Struyker-Boudier HAJ, et al. Delayed but not immediate captopril therapy improves cardiac function in conscious rats, following myocardial infarction. J Mol Cell Cardiol 1991; 23: 187-197.

4. Litwin SE, Litwin CM, Raya TE, et al. Contractility and stiffness of noninfarcted myocardium after coronary ligation in rats. Effects of chronic angiotensin converting enzyme inhibition. Circulation 1991; 83: 1028-1037.

5. Pfeffer MA, Pfeffer JM, Steinberg C, et al. Survival after an experimental myocardial infarction: beneficial effects of long-term therapy with captopril. Circulation 1985; 72: 406-412.

6. Youn TJ, Kim HS, Oh BH. Ventricular remodelling and transforming growth factor-beta $1 \mathrm{mRNA}$ expression after nontransmural myocardial infarction in rats: effects of angiotensin converting enzyme inhibition and angiotensin II type 1 receptor blockade. Basic Res Cardiol 1999; 94: 246-253.

7. Kingma JH, van Gilst WH, Peels $\mathrm{CH}$, et al. Acute intervention with captopril during thrombolysis in patients with first anterior myocardial infarction. Eur Heart J 1994; 15: 898-907.

8. Divisova J, Vavrinkova H, Tutterova M, et al. Effect of ACE inhibitor captopril and L-arginine on the metabolism and on ischaemia-reperfusion injury of the isolated rat heart. Physiol Res 2001; 50: 143-152.

9. Jugdutt BI. Nitric oxide and cardioprotection during ischaemia-reperfusion. Heart Failure Reviews 2002; 7: 391-405.

10. Ferdinandy P, Schulz R. Nitric oxide, superoxide, and peroxynitrite in myocardial ischaemia-reperfusion injury and preconditioning. Br J Pharmacol 2003; 138: 532-543.

11. Csonka C, Szilvássy Z, Fülöp F, et al. Classic preconditioning decreases the harmful accumulation of nitric oxide during ischaemia and reperfusion in rat hearts. Circulation 1999; 100: 2260-2266.

12. Hein TW, Zhang C, Wang W, et al. Ischaemia-reperfusion selectively impairs nitric oxide-mediated dilation in coronary arterioles: counteracting role of arginase. FASEB J 2003; doi 10.1096/fj.03-0115fje.

13. Sodha, NR, Boodhwani M, Clements RT, et al. Coronary microvascular dysfunction in the setting of chronic ischaemia is independent of arginase activity. Microvascular Res 2008; 75: 238-146.
14. Tabor CW, Tabor H. 1, 4-Diaminobutane (putrescine), spermidine, spermine. Ann Rev Biochem 1976; 45: 285-306.

15. Giordano E, Flamigni F, Guarnieri C, et al. Poylamines in cardiac physiology and disease. Open Heart Failure Journal 2010; 3: 25-30.

16. Schlüter K-D, Frischkopf K, Flesch M, et al. Central role for ornithine decarboxylase in $\beta$-adrenoceptor mediated hypertrophy. Cardiovasc Res 2000; 45 : 410-417.

17. Shimizu M, Irimajiri O, Nkano T, et al. Yamada H, Sasaki H, Isogai Y. Effect of captopril on isoproterenol-induced myocardial ornithine decarboxylase activity. J Mol Cell Cardiol 1991; 23: 665-670.

18. Jung C, Gonon AT, Sjoquist PO, et al. Arginase inhibition mediates cardiac protection during ischaemia-reperfusion. Cardiovasc Res 2010; 85: 147-154.

19. Fischer S, Grantzow T, Pagel JI, et al. Extracellular RNA promotes leukocyte recruitment in the vascular system by mobilising proinflammatory cytokines. Thromb Haemost11 2012; 108: 730-741.

20. Harpster MH, Bandyopadhyay S, Thomas P, et al. Earliest changes in the left ventricular transcriptosome post-myocardial infarction. Mammalian Genome 2006; 17: 701-715.

21. Gao X, Xu X, Belmadini S, et al. TNF-a contributes to endothelial dysfunction by upregulating arginase in ischaemia/reperfusion injury. Arterioscler Thromb Vasc Biol 2007; 27: 1269-1275.

22. Zhang C, Wu J, Xu X, et al. Potter BJ, Gao X. Direct relationship between levels of TNF- $\alpha$ expression and endothelial dysfunction in reperfusion injury. Basic Res Cardiol 2010; 105: 453-464.

23. Frascarelli S, Ghelardoni S, Ronca-Tzestoni S, et al. Cardioprotective effect of zofenopril in perfused rat hearts subjected to ischaemia and reperfusion. J Cardiovasc Pharmacol 2004; 43: 294-299.

24. Bencsik P, Kupai K, Giricz Z, et al. Role of iNOS and peroxynitrite-matrix metalloproteinase-2 signalling in myocardial late preconditioning in rats. Am J Physiol Heart Circ Physiol 2010; 299: H512-H518.

25. Da Costa Rebelo RM, Schreckenberg R, Schlüter K-D. Adverse cardiac remodelling in spontaneously hypertensive rats: acceleration by high aerobic exercise intensity. J Physiol 2012; 590: 5389-5400.

26. Kocsis GF, Sárközy M, Bencsik P, et al. Preconditioning protects the heart in a prolonged uremic condition. Am J Physiol Heart Circ Physiol 2012; 303: H1229-1236.

27. Livak KJ, Schmittgen TD. Analysis of relative gene expression data using realtime quantitative PCR and the 2(- $\Delta \Delta C T)$. Methods 2001; 25: 402-408.

28. Maxeiner H, Krehbiel N, Müller A, et al. New insights into paracrine mechanisms of human cardiac progenitor cells. Eur J Heart Fail 2010; 12: 730-737.

29. Canton M, Skyschally A, Menabo R, et al. Oxidative modification of tropomyosin and myocardial dysfunction following coronary microembolisation. Eur Heart J 2006; 27: 875-881.

30. Deignan JL, Livesay JC, Shantz LM, et al. Polyamine homeostasis in arginase knockout mice. Am J Physiol Cell Physiol 2007; 293: C1296-C1301.

31. Flesch M, Höper A, Dell'Italia L., et al. Activation and functional significance of the renin-angiotensin system in mice with cardiac restricted overexpression of tumor necrosis factor. Circulation 2003; 108: 598-604.

32. Mörlein C, Schreckenberg R, Schlüter K-D. Basal ornithine decarboxylase activity modifies apoptotic and hypertrophic marker expression in post-ischaemic hearts. Open Heart Failure J 2010; 3: 31-37. 